\title{
The Shape of the Magnetic Fluid Surface above a Magnetizable Sphere in a Uniform Magnetic Field
}

\author{
V. G. Bashtovoi ${ }^{a}$, A. A. Motsar ${ }^{a}$, V. A. Naletova ${ }^{b}$, and A. G. Reks ${ }^{a}$ \\ aelarus National Technical University, pr. Nezavisimosti 65, Minsk, 220013 Belarus \\ ${ }^{b}$ Research Institute of Mechanics, Moscow State University, Michurinskii pr. 1, Moscow, 119192 Russia \\ e-mail: alexfx20@yandex.ru \\ Received June 26, 2014
}

\begin{abstract}
The shape of the free surface of a magnetic fluid above a spherical ferromagnetic body immersed in it in a uniform magnetic field is investigated experimentally. The effect of the direction and magnitude of the magnetic field on the deformation characteristics of the free surface of the magnetic fluid with various magnetic properties and geometrical parameters is established.
\end{abstract}

DOI: $10.1134 / \mathrm{S} 1063784215100060$

\section{INTRODUCTION}

The interest in studying the shape of the magnetic fluid surface is primarily due to its possible practical application in some device, associated with the ability of a magnetic fluid to change the shape of its free surface under the action of a magnetic field. In particular, this property makes it possible to control heat and mass transfer during hardening of solids in a magnetic fluid [1] and to use magnetic fluids in various switches and valves.

The introduction of a magnetizable body into a uniform magnetic field leads to its distortion and the emergence of local nonuniformities. If the body is in a magnetic fluid, the field nonuniformities caused by distortions may change the shape of its free surface. It was found in [2] that a bounded volume of a magnetic fluid is distributed irregularly on a spherical steel body in a uniform magnetic field.

The problem of the shape of a magnetic fluid with bodies of various shapes immersed in it was considered in a number of publications. Theoretical and experimental investigations of deformation of the magnetic fluid surface with a magnetizable cylinder immersed into it under the action of an external uniform magnetic field were carried out in $[3,4]$. The deformation of the magnetic fluid surface by a magnetizable sphere in a uniform magnetic field was considered in [5-7]; the conformity between the theoretical and experimental results was established in [7].

Here, we report on the results of investigations revealing some peculiarities in the formation of the free surface of magnetic fluids of various magnetizations around a spherical ferromagnetic body, which continue the studies started in [6].

\section{EXPERIMENTAL SETUP}

In the experiment, the cuvette with a magnetic fluid and with a steel sphere immersed in it was placed into the uniform magnetic field produced by Helmholtz coils. The cuvette had the shape of a parallelepiped with a size of $81 \times 82 \mathrm{~mm}$. The diameter of the steel sphere was $6 \mathrm{~mm}$. The amplitude of height variation $\Delta h$ of the free surface above the body was measured by a micrometer with a thin magnetic needle and was photographed. For the origin of coordinates, we took the point on the surface of the fluid in zero magnetic field, when its entire surface was flat. The observation of the shape of the magnetic fluid surface was carried out for various values of uniform magnetic field strength $H$, the variation of which can be assumed to be quasi-static. The initial thickness $h$ of the layer of the magnetic fluid above the body was determined for magnetic field strength $H=0$ and was varied by filling the cuvette with different volumes of the magnetic fluid.

We considered several magnetic field directions. In the first case, the horizontal direction was parallel to the fluid surface. In the second case, the magnetic field vector formed an angle of $30^{\circ}$ with the horizontal. In the third case, the direction of the field was vertical and perpendicular to the initially flat equilibrium surface of the magnetic fluid.

In our experiments, we used magnetic fluids based on MMTr-10 and MMTr-44 transformer oil and MK-27 and MK-51 kerosene (the properties of these fluids are given in the table). 
Properties of fluids

\begin{tabular}{l|c|c}
\hline \multicolumn{1}{c|}{ Properties of fluids } & MMTr-44 & MMTr-10 \\
\hline \multicolumn{1}{c|}{ Fluid base } & \multicolumn{2}{|c}{ Transformer oil } \\
\hline $\begin{array}{l}\text { Saturation magnetization } M_{s}, \\
\mathrm{kA} / \mathrm{m}\end{array}$ & 43.8 & 9.6 \\
Initial magnetic susceptibility $\chi_{0}$ & 2.7 & 0.1 \\
Density $\rho_{f}, \mathrm{~kg} / \mathrm{m}^{3}$ & 1400 & 1115 \\
Surface tension $\sigma, \mathrm{mN} / \mathrm{m}$ & 28 & 30 \\
Wetting angle of the cuvette $\theta_{w}$, & 46 & 31 \\
deg & & \\
Wetting angle of the body $\theta_{c}$, deg & 46 & 31 \\
\hline
\end{tabular}

\section{HORIZONTAL MAGNETIC FIELD}

In an external uniform magnetic field, the shape of the fluid surface was determined by the existence of regions of local nonuniformity of the field around the sphere. In the case of horizontal direction of the field, its distortion occurs so that regions with the maximal value of the field are formed at the "end" faces of the sphere relative to the field, while regions with the minimal value of the field are formed at the "lateral" faces.

If the magnetizable sphere is partly immersed into a magnetic fluid, the field distortions induced by it lead to the flow of the fluid to the region with the maximal field strength (end faces of the sphere). The level of liquid in this case falls in the regions of lateral faces of the sphere, where the field is weaker. This is illustrated by a set of photographs in Fig. 1.

The direction of the horizontal field is indicated by an arrow in the figure. In zero field, the fluid is distrib- uted axisymmetrically around the body (Fig. 1a), and its surface is deformed insignificantly due to wetting of the ball surface. In a magnetic field, the surface of the fluid is deformed so that it flows to the ends regions of the sphere and forms a curvilinear surface of an intricate shape (Fig. 1b). On the lateral surface of the sphere, a central annular segment not covered with the fluid appears, and in this region dips are formed on the fluid surface. With increasing field strength, the deformation amplitude increases, as well as the size of the dips at the lateral surfaces (Fig. 1c).

If the depth of the layer of the fluid is equal to the diameter of the sphere, and the thickness of the layer of the magnetic fluid above the body is $h=0$, even in small fields of about $1 \mathrm{kA} / \mathrm{m}$, a dip appears above the sphere with the shape asymmetric relative to its central axis (Fig. 2a). In the direction of the field, the fluid flows onto the end regions of the sphere, leaving the center of its top open. In the transverse direction, in the lateral regions of the sphere, zones with a lower level of the fluid are formed. Upon a further increase in the field, the deformation of the surface becomes stronger, and the dip acquires a more complex shape (Fig. 2b). As in the case of the sphere coated with the fluid by half, a zone free of fluid is formed near the central transverse segment.

If the sphere is submerged so that a layer of the magnetic fluid of thickness $h>0$ is formed above it, an asymmetric dip appears in a horizontal magnetic field like in the previous case. An increase in the field strength increases the amplitude of deformation $\Delta h$ of the free surface of the fluid above the body.

The type of deformation of the surface and the rate of variation of its deformation amplitude $\Delta h$ upon a change in the field strength depends on the magnetic (a)

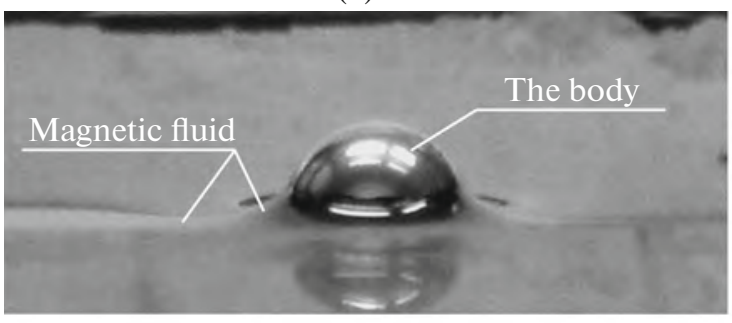

(b)

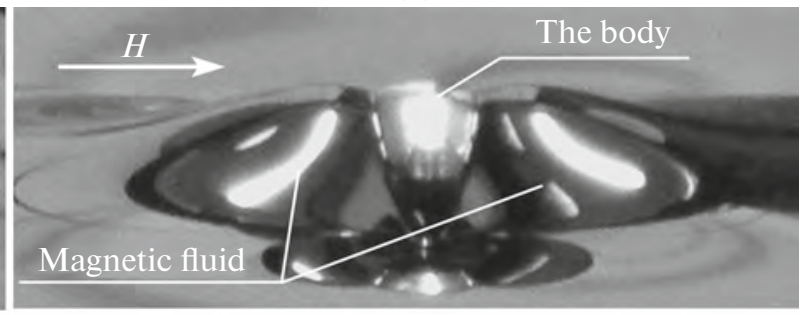

(c)

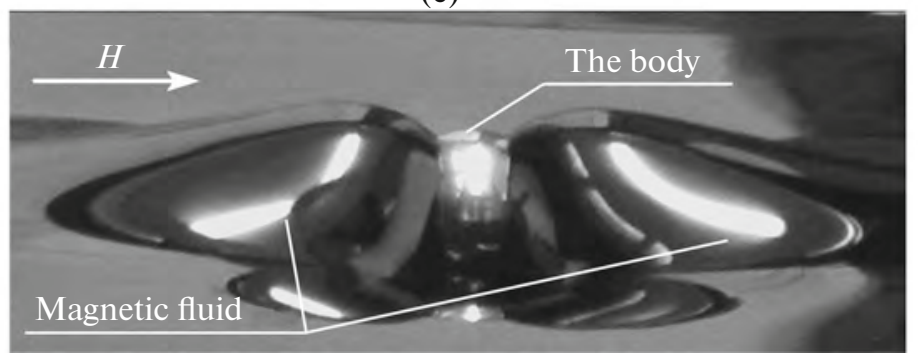

Fig. 1. Shape of the free surface of a magnetic fluid with partial submergence of the sphere into it; $H, \mathrm{kA} / \mathrm{m}$ : (a) 0 , (b) 9.7 , and (c) 23.9 . 
(a)

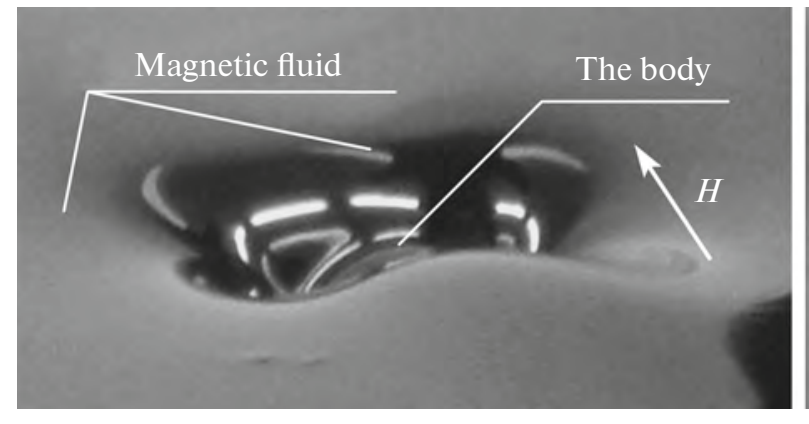

(b)

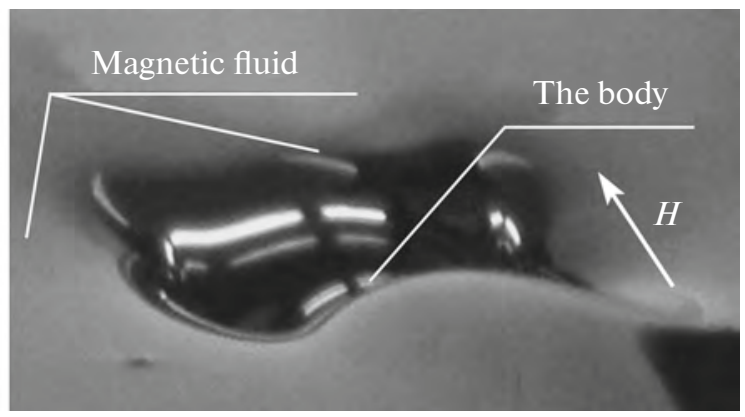

Fig. 2. Shape of the free surface of a magnetic fluid with complete submergence of the sphere into it; $H$, kA/m: (a) 10.2 and (b) 23.9 .

properties of the fluid and on initial thickness $h$ of the layer of the fluid above the sphere.

For small layer thicknesses, the deformation of the surface is smooth: the amplitude of the dip increases monotonically with the field until it becomes equal to the thickness of the fluid layer, and the top of the sphere is open in this case. With increasing thickness of the fluid layer above the sphere, the rate of variation of the deformation amplitude decreases with increasing field (Fig. 3, curves for MMTr-44 fluid, layer thickness is 2.1 and $3 \mathrm{~mm}$ ). This is due to the fact that for a large thickness, the surface of the fluid is located in the region at the larger distance from the sphere, where the magnetic field distortions are weaker.

It is found that there exists a certain thickness of the fluid layer above the sphere, above which an abrupt rupture of the fluid layer down to the sphere surface may occur. For such thicknesses of the layer, there exists the critical value of magnetic field strength $H_{\text {crr1 }}$ at which the amplitude of the dip on the fluid surface

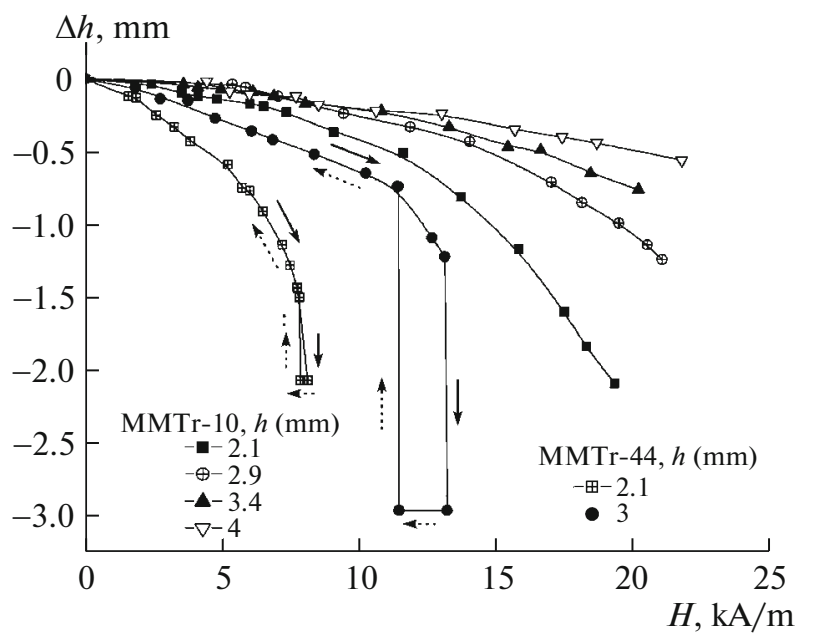

Fig. 3. Dependence of the amplitude of deformation of the magnetic fluid surface on the external magnetic field strength. becomes jumpwise equal to the thickness of the layer (see Fig. 3, the curve for MMTr-4 fluid, thickness of the layer is $3 \mathrm{~mm}$ ). A vertical channel of an intricate shape changing with increasing field is formed above the sphere.

For the opposite direction of field variation, the channel above the sphere disappears also abruptly in fields $H_{\text {crr2 } 2}$ smaller than $H_{\text {crr1 }}$. The solid arrow in Fig. 3 for MMTr-44 fluid shows the variation of deformation amplitude $\Delta h$ upon an increase in the field, while the dotted arrow shows the variation upon a decrease in the field. In the range of fields smaller than $H_{\text {crr2 }}$, the dependences of the deformation amplitude in the forward and reverse directions of the field variation coincide (i.e., there is now hysteresis of the shape). The shape hysteresis is not observed for small thicknesses of the fluid layer above the sphere either.

Thus, it can be seen that in the range of fields from $H_{\text {crr2 }}$ to $H_{\text {crr1 }}$, the hysteresis in the shape of the free surface is observed, and the shape depends on the direction of field variation.

With increasing thickness $h$, critical field $H_{\text {crr1 }}$ increases, as well as the difference between critical fields $H_{\text {crr1 }}$ and $H_{\text {crr2 } 2 \text { [7]. }}$

Figure 3 shows for comparison the characteristics of deformation for fluids with different magnetizations, MMTe-44 $\left(M_{s}=43.8 \mathrm{kA} / \mathrm{m}\right)$ and MMTr-10 $\left(M_{s}=9.6 \mathrm{kA} / \mathrm{m}\right)$. The deformation of the surface occurs under the action of bulk magnetic force $\mu_{0} M \operatorname{grad} H$, which is proportional to magnetization $M$ of the fluid. For this reason, for the same magnetic field strength, the amplitude of deformation of the surface is smaller for the fluid with a lower magnetization.

For MMTr-10 fluid with a small magnetization, in contrast to MMTr-44 fluid with stronger magnetic properties, no jumpwise rupture of the layer of the fluid is observed in the range of magnetic fields under investigation up to $30 \mathrm{kA} / \mathrm{m}$, and there is no hysteresis of the shape of the surface. The amplitude of deformation of the free surface of the fluid varies monotoni- 

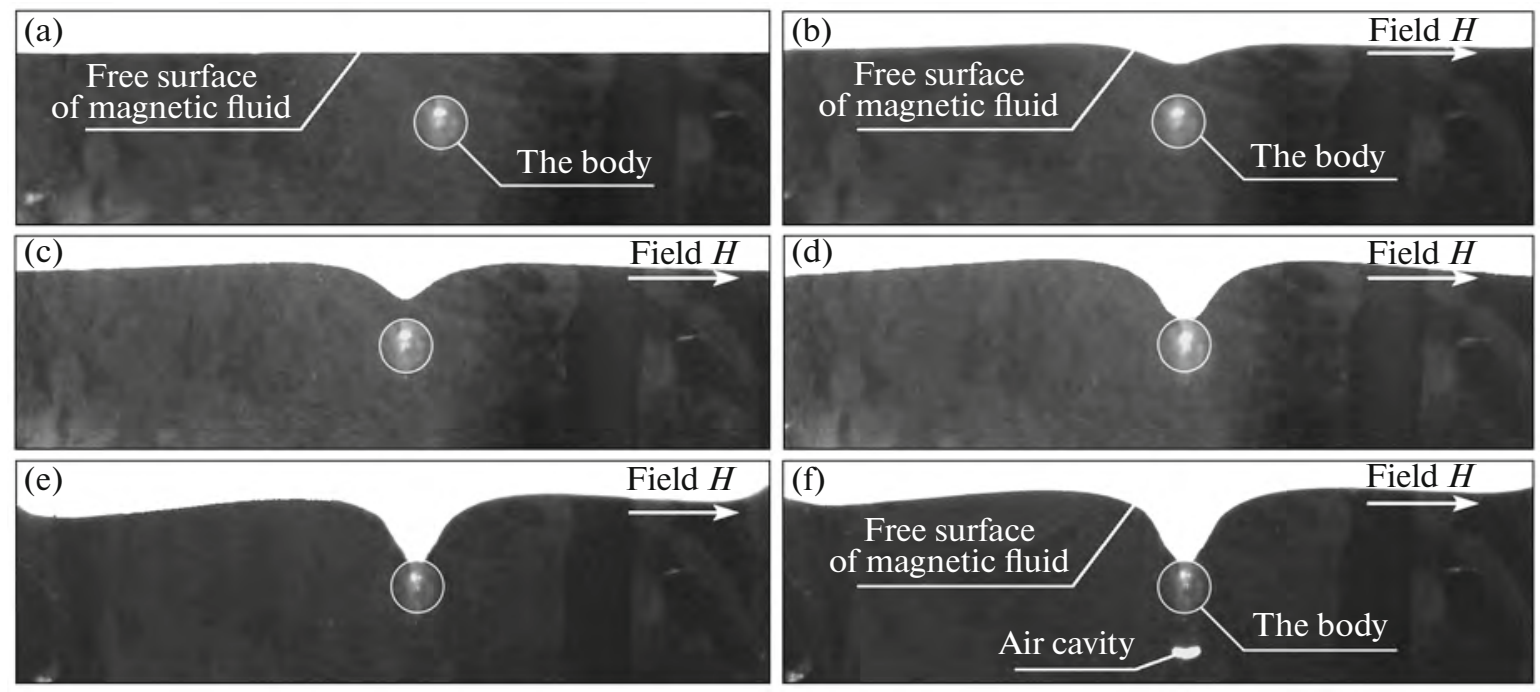

Fig. 4. Shape of the free surface of a magnetic fluid in a horizontal magnetic field for MK-27 magnetic fluid; $H$, kA/m: (a) 0 , (b) 5.7, (c) 8.41, and (d) 19.9; for MK-51 magnetic fluid: (e) 16; (f) 21.8.
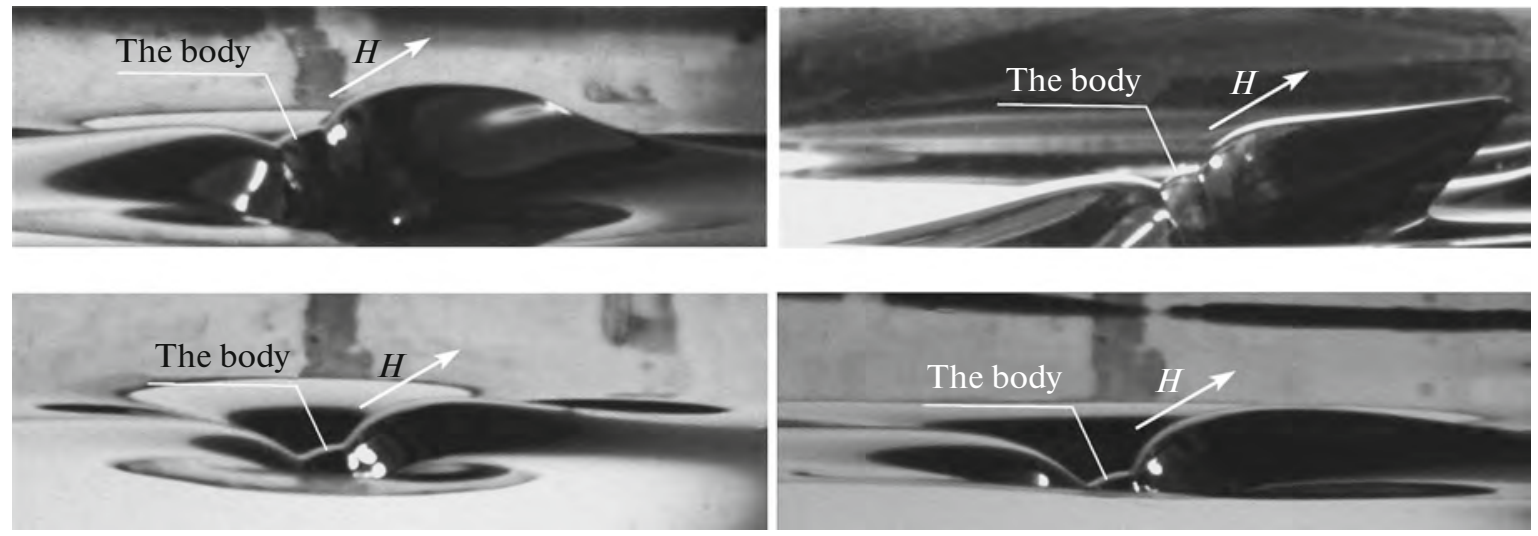

Fig. 5. Shape of the free surface of a magnetic fluid in a tilted magnetic field $H, \mathrm{kA} / \mathrm{m}$ : (a) 16.7, (b) 23, (c) 13.3 , and (d) 21.7 .

cally with the field. Upon an increase in the thickness of the fluid layer, the rate of variation of the deformation amplitude decreases.

To visualize the variation of the shape of the free surface in the horizontal field, we performed a set of experiments with a magnetizable sphere placed into a narrow slit with a size of $6 \times 54 \mathrm{~mm}$. The sphere is at equal distances from the faces of the slit; for this reason, in contrast to the case investigated in [6], the fluid was not only above the sphere, but also under it. Figure 4 shows photographs for fluids based on MK-27 and MK-51 kerosene with the saturation magnetizations of 27 and $51 \mathrm{kA} / \mathrm{m}$, respectively. For the situations depicted in the figure, the magnetic field is directed along the slit.

Figures $4 a-4 d$ illustrate the shape of the free surface of MK-27 fluid, while Figs. 4e and $4 \mathrm{f}$ show it for MK-51 fluid with stronger magnetic properties. Figures $4 \mathrm{a}-4 \mathrm{c}$ illustrate the case with fields $H<H_{\text {crr } 1}$ prior to rupture of the fluid surface above the sphere. Photographs in Figs. $4 d-4 f$ show the rupture of the surface of the magnetic fluid in fields exceeding the critical value $\left(H>H_{\text {crr1 } 1}\right)$.

It was found that in fields exceeding $H_{\text {crr } 1}$, after the formation of an air channel above the sphere, further deformation of the surface occurs with the formation of a vertical annular air cavity around the sphere. This cavity can clearly be seen in Fig. 4f.

\section{TILTED MAGNETIC FIELD}

In a tilted magnetic field, we considered the partial and complete submergence of a magnetizable sphere into a magnetic fluid. In our experiment, the tilt angle of the magnetic field vector to the horizontal was $30^{\circ}$.

The shapes of the fluid surface in a tilted magnetic field are shown in Fig. 5. Figures 5a and 5b show the 


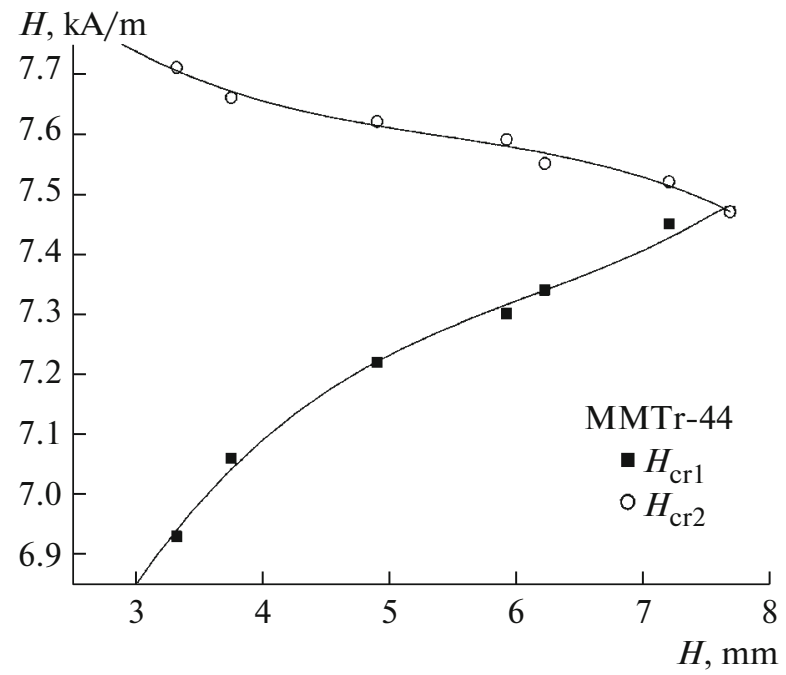

Fig. 6. Dependence of critical fields for MMTr- 44 on the thickness of the layer of the fluid above the body.

case with a partial submergence of the sphere into the fluid. As in the case with the horizontal field, the fluid flows to the sphere in the end regions of its surface where the magnetic field is stronger. On the one side of the sphere, the level of the fluid is higher and resembles the wave crest.

With increasing field strength $H$, this region of the surface is transformed into a tilted conical peak (Fig. 5b). Due to the action of the force of gravity, the angle of inclination of the peak to the horizontal is slightly smaller than the tilt angle of field $H$. In the region of lateral surfaces of the sphere, dips with an intricate shape are formed on fluid surface.

When the body is completely covered with the fluid, the surface above the sphere is deformed upon the application of the field, and at a certain value of field strength $H$, the top of the sphere becomes open, and the fluid flows to the sphere along the field in the same way as in the previous case (Figs. 5c, 5d). With increasing field, the deformation of the surface increases in the direction of the field. In the range of fields under investigation up to $30 \mathrm{kA} / \mathrm{m}$, no conical peak was formed along the field.

\section{VERTICAL MAGNETIC FIELD}

In a vertical uniform magnetic field, the variation of the shape of the fluid depends on the depth of submergence of the sphere into the magnetic fluid.

If the sphere is half-submerged into the magnetic fluid, the application of the external uniform magnetic field leads to the formation of an annular depression cone is formed around the sphere with a depth increasing with the field; the height of wetting of the sphere by the fluid changes thereby.
When the layer of the fluid covers the sphere completely, the vertical field leads to the emergence of a protrusion of the fluid above the sphere, the deformation amplitude $\Delta h$ and the shape of the protrusion depending on field strength $H$. For example, it was established in [6] that there exists $H_{\mathrm{cr} 1}$ at which the shape of the protrusion is abruptly transformed from a smooth spherical hill to a conical vertical peak. In this case, the rate of variation of the deformation amplitude sharply increases with the field. The critical field $H_{\text {crl }}$ increases with thickness $h$ of the layer of the fluid. The characteristics of deformation of the fluid surface in the vertical field are described in $[6,7]$.

At a certain critical value of field $H_{\mathrm{cr} 2}$, an instability manifested in the jumpwise formation of a set of peaks over the entire surface develops in the region of the fluid surface at large distances from the sphere. It was established earlier [8] that critical field $H_{\text {cr2 }}$ is determined by the depth of the fluid layer. It decreases monotonically with increasing depth of the layer. In the range of fields $H>H_{\mathrm{cr} 2}$, the rate of variation of the deformation amplitude above the sphere decreases upon an increase in the field.

With increasing depth of the layer of the fluid, thickness $h$ of the layer above the sphere increases. The surface of the liquid is now at a larger distance from the sphere; accordingly, field nonuniformities appearing in this case become smaller. As a result, the influence of the presence of the sphere in the fluid on the shape of its surface becomes weaker, and critical fields $H_{\text {cr1 }}$ and $H_{\mathrm{cr} 2}$ become closer and tend to the critical field for a thick layer of the fluid.

Figure 6 shows the experimental dependences of the critical fields for the surface of MMTr-44 magnetic fluid above the sphere and at a large distance from it on thickness $h$ of the layer of the fluid.

\section{CONCLUSIONS}

The results of the investigation show that zones of local inhomogeneity are formed around a spherical magnetizable body in a uniform magnetic field as a result of field redistribution. When this body is placed onto a plane layer of a magnetic fluid in a uniform magnetic field, the flat surface of the fluid is deformed. The characteristics of deformation of the surface are determined by the magnitude and orientation of the magnetic field.

\section{ACKNOWLEDGMENTS}

This study was supported by the Foundation for Basic Research of the Belarus Republic and the Russian Foundation for Basic Research (project no. 1401-90003). 


\section{REFERENCES}

1. V. V. Gogosov, V. V. Kiryushin, and A. Ya. Simonovskii, Magnetohydrodynamics 30, 163 (1994).

2. V. V. Gogosov, V. A. Naletova, and G. A. Shaposhnikova, Itogi Nauki Tekh., Ser.: Mekh. Zhidk. Gaza 16, 76 (1981).

3. K. Zimmermann, V. A. Naletova, I. Zeidis, V. A. Turkov, D. A. Pelevina, V. Bohm, and J. Popp, Magnetohydrodynamics 44, 175 (2008).

4. K. Zimmermann, I. Zeidis, V. A. Naletova, V. A. Turkov, J. Popp, A. V. Rozin, and D. A. Pelevina, J. Magn. Magn. Mater. 324, 1253 (2012).
5. V. A. Naletova, D. A. Pelevina, and V. A. Turkov, Fluid Dyn. 44, 797 (2009).

6. V. Bashtovoi, A. Motsar, V. Naletova, A. Reks, and D. Pelevina, Magnetohydrodynamics 49, 592 (2013).

7. D. Pelevina, V. Naletova, V. Bashtovoi, A. Motsar,and A. Reks, Magnetohydrodynamics 50, 83 (2014).

8. V. G. Bashtovoi, B. M. Berkovskii, and A. N. Vislovich, Introduction to Thermomechanics of Magnetic Liquids (IVTAN SSSR, Moscow, 1985).

Translated by N. Wadhwa 